\section{Two New Macrolides Produced by Streptomyces sp. CS}

\author{
Chunhua Lu and Yuemao Shen* \\ The State Key laboratory of Phytochemistry and \\ Plant Resources in West China, Kunming Institute of Botany, \\ Chinese Academy of Sciences, \\ Kunming 650204, P. R. China \\ (Received for publication February 23, 2004)
}

A strong antifungal and antitumor agent, 24-demethylbafilomycin $\mathrm{C}_{1}(\mathbf{1})$ has been isolated from Streptomyces sp. $\mathrm{CS}^{11}$. The structure of $\mathbf{1}$ is different from those of bafilomycins at the replacement of the isopropyl group with ethyl group at C-23, and different from hygrolidins at C-2 substitution and the orientations of the substituents at C-21, $\mathrm{C}-22$ and $\mathrm{C}-23$ in the pyranose ring $^{2 \sim 5)}$. Therefore it belongs to a new subfamily of 16 -membered macrolides. The isolation of $\mathbf{1}$ from this strain in previous work encouraged us to search for this type of metabolites further, which led to the isolation of compound $\mathbf{1}$ and two new bafilomycin derivatives $\mathbf{2}$ and $\mathbf{3}$. Their structures were elucidated based on the NMR experiments.

\section{Experimental}

Fermentation and Extraction

A stock of Streptomyces sp. CS was cultured on ISP2 agar plates at $28^{\circ} \mathrm{C}$ for 7 days and a single colony was inoculated to a $500 \mathrm{ml}$ Erlenmeyer flask containing $50 \mathrm{ml}$ ISP2 broth as a seed medium. The flask was incubated at $28^{\circ} \mathrm{C}$ for 2 days on a rotary shaker $(180 \mathrm{rpm})$. The seed culture was inoculated onto Petri dishes containing ISP3 agar media ( $c a .20 \mathrm{ml} / \mathrm{dish}$ ). The fermentation was cultivated at $28^{\circ} \mathrm{C}$ for 7 days.

The cultured agar media (14 liters, 700 dishes) was chopped, diced and extracted with EtOAc-MeOH - AcOH $(80: 15: 5,14$ liters $)$ at room temperature for over night.
The organic solution was collected through filtration with filter paper, and the remaining agar residue was extracted several times more as described above until the filtrate colourless. The combined filtrates were concentrated under vacuum to remove organic solvents. The aqueous solution was extracted five times with chloroform to afford the No. 1 $\mathrm{CHCl}_{3}$ extract $(11.9 \mathrm{~g})$ after the removal of solvents under vacuum. After further concentration under vacuum, the aqueous phase was filtrated through filter paper to produce some crystal-like residue, which was dissolved in chloroform. After being washed with distilled water and the removal of solvents under vacuum, this chloroform solution afforded the No. $2 \mathrm{CHCl}_{3}$ extract $(6.58 \mathrm{~g})$ after the removal of solvents under vacuum. Those two portions of aqueous solutions were combined and further extracted exhaustively with chloroform to produce the No. $3 \mathrm{CHCl}_{3}$ extract $(1.09 \mathrm{~g})$ and $\mathrm{H}_{2} \mathrm{O}$ extract $(30.5 \mathrm{~g})$ after the removal of solvents under vacuum, respectively.

\section{Isolation}

The No. $2 \mathrm{CHCl}_{3}$ extract $(6.58 \mathrm{~g})$ was first chromatographed over Sephadex LH-20 (120g) and eluted with methanol. The fractions were combined according to the antifungal activities against Penicillium avellaneum UC-4376 with paper disc diffusion assay and TLC results over $\mathrm{Si}$ gel developed by petroleum ether-acetone $(5: 1$, $\mathrm{v} / \mathrm{v})$ and chrloroform - methanol $(10: 1, \mathrm{v} / \mathrm{v})$. The combined fractions (No. $8 \sim 11,2.0 \mathrm{~g}$ ) were subjected to column chromatography over $\mathrm{Si}$ gel $(40 \mathrm{~g})$ and eluted with petroleum ether-acetone $(10: 1)$ and 12 fractions were collected. Fractions $7 \sim 12$ showed strong antifungal activities. Fraction 1 (633 mg) was subjected to column chromatography over Si gel $(8 \mathrm{~g})$ eluted with petroleum ether-acetone $(10: 1)$, and the combination was monitored by TLC and followed by MPLC over reversed-phase $\mathrm{C}_{18} \mathrm{Si}$ gel $(24 \mathrm{~g})$ eluted with gradient acetone - water $(7: 3 ; 8: 2$, $\mathrm{v} / \mathrm{v})$ to afford compound $2(15 \mathrm{mg})$. Fractions $4 \sim 5$ (124 mg) were isolated with repeated MPLC over reversedphase $\mathrm{C}_{18}$ Si gel $(24 \mathrm{~g})$ eluted with acetone-water $(7: 3$, $\mathrm{v} / \mathrm{v})$, and followed with column chromatography over Si gel (6.6 g) eluted with gradient chloroform-methanol

* Corresponding author: yshen@mail.kib.ac.cn

This paper is dedicated to my mentor, Professor Dr. HEINZ G. Floss, at the University of Washington on the occasion of his 70th birthday. 
$(100: 1.5,100: 2)$ to afford $3(5 \mathrm{mg})$. Fractions $7 \sim 12$ $(570 \mathrm{mg})$ were chromatographed over reversed-phase $\mathrm{C}_{18} \mathrm{Si}$ gel $(140 \mathrm{~g})$ and eluted with methanol-water $(7: 3)$ to produce compound $\mathbf{1}(80 \mathrm{mg})$. Compounds 1 ( $10 \mathrm{mg}$ ) and $\mathbf{2}$ $(5 \mathrm{mg})$ were isolated from the No. $1 \mathrm{CHCl}_{3}$ extract through repeated column chromatography over reversed-phase $\mathrm{C}_{18}$ Si gel ( $140 \mathrm{~g})$ eluted with gradient acetone-water $(7: 3$; $8: 2$ ) and normal phase $\mathrm{Si}$ gel eluted with chloroformmethanol (10:1, 5:1, v/v) and Sephadex LH-20 eluted with methanol as well. The Rf values for compounds 1, 2 and 3 on TLC plates precoated with $\mathrm{Si}$ gel $\mathrm{F}_{254}$ (Qingdao Marine Chemical Ltd., People's Republic of China) were
0.3 (developed twice with chloroform-methanol, 10:1), 0.4 (developed twice with petroleum ether-acetone, $3: 1$ ) and 0.3 (developed with chloroform-methanol, 20:1), respectively.

\section{Biological Assays}

The paper disc diffusion assay ${ }^{6)}$ was used for measuring the antifungal activity against Penicillium avellaneum UC4376 and the protein-binding sulforhodamine (SRB) and microculture tetrozolium (MTT) method for the cytotoxicity against cancer cell ${ }^{7.8)}$.

Table 1. The NMR data for compounds 2 and $\mathbf{3}$.

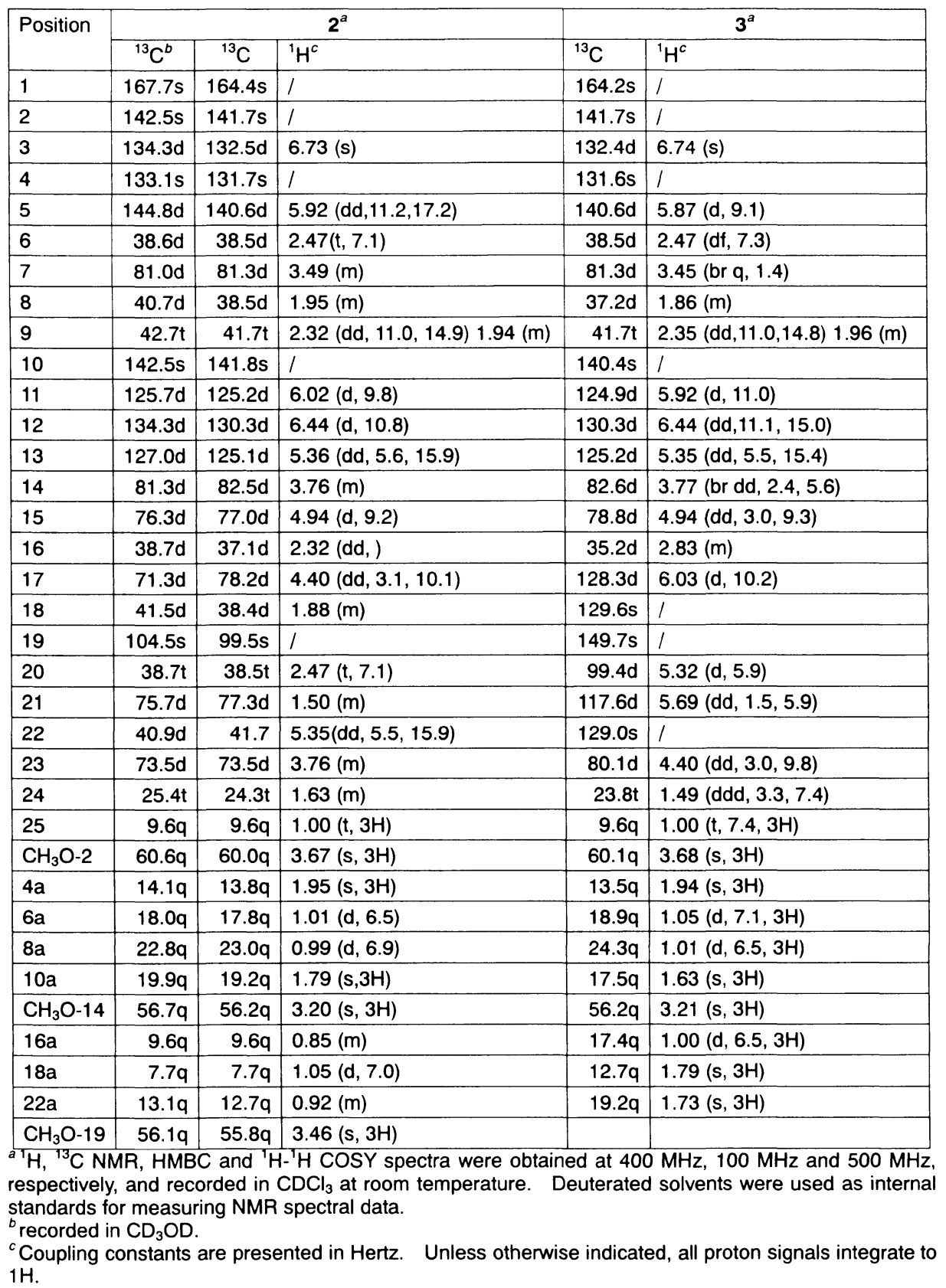




\section{Result and Discussions}

Compound 1 was determined to be 24-demethylbafilomycin $\mathrm{C}_{1}$ by comparing with the authentic sample on TLC, and the literature spectroscopic data").

Compound 2, colorless crystal, was determined to have the molecular formula of $\mathrm{C}_{35} \mathrm{H}_{58} \mathrm{O}_{9}$ based on the negative HRFABMS data $[\mathrm{m} / \mathrm{z}, 622.4070$, calcd 622.4081]. Inspection of the NMR data (proton, carbon, DEPT, HMQC and $\mathrm{HMBC}$ ) revealed a bafilomycin-type 16-membered macrolide (Table 1$)^{3)}$. The ${ }^{13} \mathrm{C}$-NMR and DEPT spectra of $\mathbf{2}$ showed thirty-five carbon signals for eight methyl, three methoxy, three methylene, sixteen methine, and five quarternary carbon atoms. According to the ${ }^{1} \mathrm{H}_{-}{ }^{13} \mathrm{C}$ longrange correlations, particularly, those of eight methyl protons with the corresponding carbons and ${ }^{1} \mathrm{H}-{ }^{1} \mathrm{H}$ COSY spectra, a bafilomycin $\mathrm{A}_{2}$ derivative was elucidated ${ }^{3)}$. The absolute configuration was determined by comparing the coupling constant with those of bafilomycin $\mathrm{C}_{1}{ }^{9}$. Therefore, compound $\mathbf{2}$ was determined to be 24-demethylbafilomycin $\mathrm{A}_{2}$. In contrast to the ${ }^{1} \mathrm{H}$ NMR data in MeOD (methanol- $d_{4}$ ) as ill-defined, compound $\mathbf{2}$ gave crisp, clean ${ }^{1} \mathrm{H}$ NMR spectra in $\mathrm{CDCl}_{3}$ at ambient temperature. But compound 2 has weak stability in $\mathrm{CDCl}_{3}$, and after 18 hours the ${ }^{13} \mathrm{C}$ NMR spectra showed signals in pairs. HMQC and $\mathrm{HMBC}$ experiments unambiguously assigned ${ }^{13} \mathrm{C}$ NMR data for C-18 to C-25 of $\mathbf{2 a}$ and $\mathbf{2 b}$ (Figure 1), determining the structures of two open chain ketones $\mathbf{2 a}$ and $\mathbf{2} \mathbf{b}$ which probably produced through free-radical reactions.

Compound 3 was determined to have the molecular formula $\mathrm{C}_{34} \mathrm{H}_{50} \mathrm{O}_{6}$ based on HRESIMS data $\left(\mathrm{C}_{34} \mathrm{H}_{50} \mathrm{O}_{6} \mathrm{Na}\right.$, $\mathrm{m} / z$ 577.3484, calcd 577.3505), and showed similar pattern as compound 2 in the ${ }^{1} \mathrm{H}$ and ${ }^{13} \mathrm{C}$ NMR spectra except for the signals for $\mathrm{C}-16, \mathrm{C}-17, \mathrm{C}-18, \mathrm{C}-18 \mathrm{a}$ and the pyranose ring (Table 1). The two protons at $\delta 5.32(\mathrm{H}-20)$ and 5.69 (H-21) indicated the presence of carbon-carbon double bond in the pyranose ring and determined the ${ }^{13} \mathrm{C}$ NMR assignments for this moiety with the aid of HMBC and HMQC experiments. Thus the structure of $\mathbf{3}$ was deduced to be dehydrated form of compound 2 (Figure 1), and fully supported by extensive interpretation of 2D NMR experiments. The orientation of the substituent at C-23 was determined to be equatorial by comparing with compound 1 and 2.

Compound 1 showed strong antifungal and potent cytotoxicity against human cell lines" ${ }^{1)}$. Compounds $\mathbf{2}$ and $\mathbf{3}$ showed no inhibitory activity against Penicillium avellaneum UC-4376 at $200 \mu \mathrm{g} /$ disc in paper disc diffusion assays. Compound $\mathbf{2}$ showed strong antitumor activities against P388 and A-549 cell lines with $\mathrm{IC}_{50} 1.13$ and
Fig. 1. The structures of compounds 1, 2, 2a, 2b and 3 , and the selected ${ }^{13} \mathrm{C}$ NMR spectral data for $\mathbf{2 a}$ and $\mathbf{2 b}$
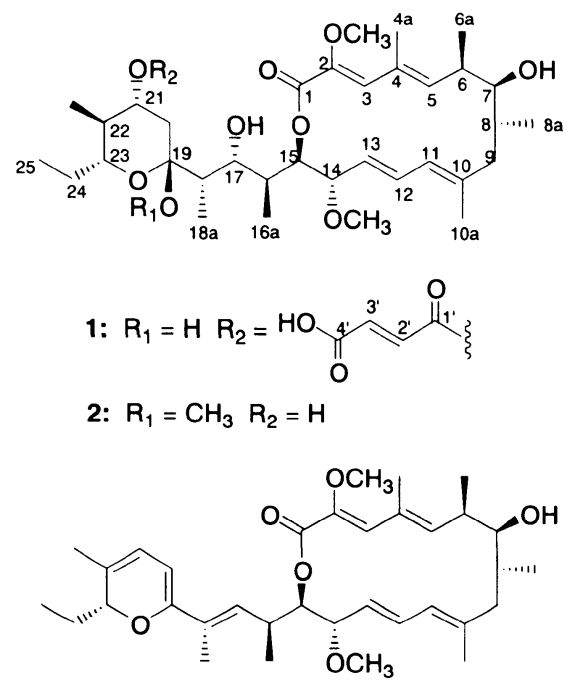

3

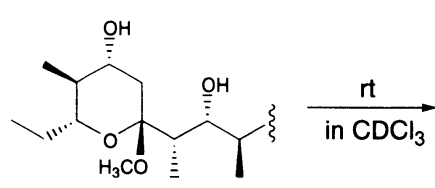

2

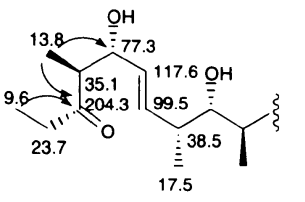

$2 a$

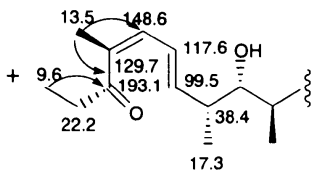

2b
$0.01 \mu \mathrm{M}$, respectively.

Previous studies showed that the substitution change at $\mathrm{C}-21-\mathrm{OH}$ in bafilomycins and hygrolidins was the main effect on their activity. But compound 2 showed almost the same cytotoxic activities as $\mathbf{1}$ and no antifungal activities, which suggested that the substitute of fumaric acid at C-21 does not affect the anticancer activity. Structural modification at C-21 would give valuable structure-activity information.

\section{Acknowledgements}

This work was partially supported by the National Science Fund for Distinguished Young Scholars to Y.-M. SHEN (30325044), the National Natural Science Foundation of China (30070007), Chinese Academy of Sciences (KSCX2-SW-313) and Natural Science Foundation of Yunnan Province (99B0017G). 


\section{Reference}

1) LU, C.-H. \& Y.-M. SHEN: A new macrolide antibiotic with antitumor activity produced by Streptomyces sp. CS, a commensal microbe of Maytenus hookeri. J. Antibiotics 56: 415 418, 2003

2) Hensens, O. D.; R. L. Monaghan, L. Huang \& G. A. AlbERS-SChONBERG: Structure of the sodium and potassium ion activated adenosinetriphosphatase inhibitor L-680,110. J. Am. Chem. Soc. 105: 3672 3679,1983

3) Werner, G.; H. Hagenmaier, H. Drautz, A. BUMgarTNER \& H. ZÄHNER: Metabolic products of microorganisms. $224^{\dagger}$ Bafilomycins, a new group of macrolide antibiotics: production, isolation, chemical structure and biological activity. J. Antibiotics 37: $110 \sim 117,1984$

4) Corey, E. J. \& J. W. Ponder: Stereochemistry of the hygrolidins. Tetrahedron Lett. 25: 4325 4328, 1984

5) Seto, H.; H. Akao, K. Furihata \& N. Otake: The structure of a new antibiotic, hygrolidin. Tetrahedron
Lett. 23: 2667 2670, 1982

6) Espinel-Ingroff, A. T.; M. A. White \& Pfaller: Antifungal agents and susceptibility tests. In: Manual of Clinical Microbiology, 7th Edn. (Murray P. R. Ed.). pp. 1640 1652. American Society for Microbiology. Washington DC, 1999

7) Mosmann, F.: Rapid calorimetric assay for cellular growth and survival: application to proliferation and cytotoxicity assay. J. Immunol. Methods 65: 55 63, 1983

8) Rubinstein, L. V.; R. H. Shoemaker, K. D. Paull, R. M. Simon, S. Tosini, P. Skehan, D. A. Scudiero, A. Monks \& M. R. BoYD: Comparison of in vitro anticancer-drugscreening data generated with a tetrazolium assay versus a protein assay against a diverse panel of human tumor cell lines. J. Natl. Can. Inst. 82: 1113 1118, 1990

9) O’Shea, M. G.; R. W. Rickards, J. M. Rothschild \& E. LACEY: Absolute configurations of macrolide antibiotics of the bafilomycin and leucanicidin groups. J. Antibiotics 50: $1073 \sim 1077,1997$ 